\title{
Development of DFT Based MATLAB and LABVIEW Models for Phasor Measurements
}

\author{
Alok Jain and M. K. Verma
}

\begin{abstract}
With the advent of Phasor Measurement Units (PMUs), it has become quite possible to monitor and control power system networks in real time. PMUs are able to provide time stamped measurements of voltage and current phasors using Global Positioning System (GPS) satellites, in microseconds. Different phasor estimation techniques such as Fast Fourier transform (FFT), Discrete Fourier transform (DFT) may be used by PMUs to estimate phasors. In this paper, MATLAB and LABVIEW models of PMU have been developed to estimate phasors. Proposed models consider use of DFT using non-recursive as well as recursive algorithms. MATLAB based model has been shown to take less time compared to LABVIEW based model.
\end{abstract}

Index Terms-Discrete Fourier Transform (DFT), Phasor measurement unit (PMU), wide area monitoring system (WAMS).

\section{INTRODUCTION}

In the Wide area Monitoring System (WAMS), it has been possible to continuously monitor the health of power system networks using synchrophasor technology. A Wide Area Monitoring System (WAMS) consists of geographically dispersed Phasor Measurement Units (PMUs) which are able to perform time stamped phasor measurements within a microsecond through Global Positioning system [1]. Role of PMU and WAMS technology in power quality estimation, protection and control of power system networks has been presented [2], [3]. Discrete Fourier Transform (DFT) based LABVIEW model of PMU using non-recursive and recursive algorithms has been developed [4]. Possibility of PMU placements in distribution systems has been considered [5]. In [6], feasibility of PMUs in smart grid architecture has been presented. A MATLAB based PMU simulator for state estimation in a three-phase network using greedy algorithm and integer programming has been presented [7], [8]. The main computational algorithms involved in the phasor measurement process are illustrated using a MATLAB based PMU simulator [9]. A novel method of incorporating the phasor measurements and the results of the traditional state estimator in a post-processing linear estimator has been proposed [10]. The performances of a PMU prototype based on a synchrophasor estimation algorithm conceived for the monitoring of active distribution networks, as well as its experimental application during some intentional islanding and reconnection tests of an urban medium voltage power network has been described [11]. Development of test

Manuscript received August 23, 2016; revised November 14, 2016.

Alok Jain and M. K. Verma are with the Department of Electrical Engineering, Indian Institute of Technology, (BHU), Varanasi 221005, India (e-mail: alok.rs.eee14@itbhu.ac.in,mkverma.eee@iitbhu.ac.in). platforms for PMU-based wide-area monitoring and control applications have been proposed [12]-[14].

In this paper, a Discrete Fourier Transform (DFT) based non-recursive and recursive algorithms has been used for the estimation of phasors through simulation which is carried using MATLAB and LABVIEW softwares. In order to test efficiency of MATLAB and LABVIEW based phasor estimation models, a test sinusoid has been estimated using non-recursive and recursive algorithms. Results of two models have been compared in estimation of test sinusoid.

\section{MEthodology FOR PHASORS Estimation}

Present work estimates phasors using DFT that is a method in which Fourier transform of a number of samples is calculated taken from an input signal $x(t)$. The Fourier transform is calculated at discrete steps in the frequency domain.

Consider a pure sinusoidal quantity given by-

$$
x(t)=\sqrt{ } 2 X \operatorname{Sin}(\omega t+\varphi)
$$

$\omega$ is the frequency of the signal in radian per second, and $\varphi$ is the phase angle in radians.

Considering (1) and assuming that $x(t)$ is sampled $N$ times per cycle,

$$
x(k)=\sqrt{ } 2 X \operatorname{Sin}\left(\frac{2 \pi}{N} k+\varphi\right)
$$

The DFT of $x(k)$ contains the fundamental frequency component given by-

$$
X=\frac{2}{N} \sum_{k=0}^{N-1} x(k) \cdot e^{\frac{-j 2 \pi k}{N}}=X_{c^{-}} j X_{S}
$$

where,

$$
X_{c}=\frac{2}{N} \sum_{k=0}^{N-1} x(k) \cdot \operatorname{Cos}\left(\frac{2 \pi k}{N}\right)
$$

and,

$$
X_{s}=\frac{2}{N} \sum_{k=0}^{N-1} x(k) . \operatorname{Sin}\left(\frac{2 \pi k}{N}\right)
$$

Considering $(N-1)$ sample as the last sample for the phase estimation, the phasor $X^{N-1}$ is given by-

$$
X^{N-1}=X_{c}^{N-1}-j X_{s}^{N-1}=X[\operatorname{Cos} \varphi+j \operatorname{Sin} \varphi]=X e^{j \varphi}
$$

In (6), the $\varphi$ represents the angle between the time of the 
first sample and the peak of the input signal.

\section{A. Non-Recursive Phasor Computation [1]}

Considering the data sample for the $r^{\text {th }}$ window-

$$
\begin{gathered}
X_{c}{ }^{(r)}=\frac{2}{N} \sum_{k=0}^{N-1} x(k+r-1) \cdot \operatorname{Cos}\left(\frac{2 \pi k}{N}\right) \\
\text { and, } X_{s}^{(r)}=\frac{2}{N} \sum_{k=0}^{N-1} x(k+r-1) \cdot \operatorname{Sin}\left(\frac{2 \pi k}{N}\right) \\
X^{*}{ }^{(r)}=\frac{1}{\sqrt{2}} X_{s}^{(r)}+j X_{c}^{(r)}=X^{*(r-1)} \cdot e^{\frac{j 2 \pi k}{N}}
\end{gathered}
$$

Phasor estimation using (9) represents non-recursive algorithm as it requires $2 N$ multiplications and $2(N-1)$ additions. While progressing of one data to next window, only one sample $x_{0}$ is discarded and sample $x_{N}$ is added to the data set. As the phasor calculations are performed fresh for each window without using any data from the earlier estimates, this algorithm is known as a non-recursive algorithm.

\section{B. Recursive Phasor Computation [1]}

In recursive computation, an update is to be made in old data phasor that determines the value of new phasor. Superscripts 'new' signify computation from data window 2 and 'old' signify computation from data window 1 . Consider the sinusoid,

$$
x(t)=\sqrt{ } 2 X \operatorname{Sin}\left(\omega t+\varphi+\frac{2 \pi}{N}\right)
$$

where, $N=$ number of samples taken in data window. With recursive algorithm, phasor using new data window 2 may be estimated using-

$$
X^{*(n e w)}=X e^{j\left(\varphi+\frac{2 \pi}{N}\right)}=X^{*(o l d)} e^{j\left(\frac{2 \pi}{N}\right)}
$$

In general, the $r^{\text {th }}$ phasor is computed from the $(r-1)^{\text {th }}$ phasor by-

$$
X^{*(r)}=X^{*(r-1)}+j^{\frac{1}{\sqrt{2}} \cdot \frac{2}{N}}\left(X_{N+r^{-}} X_{r}\right) \cdot e^{\frac{-j 2 \pi}{N}(r-1)}
$$

In the recursive algorithm, only two multiplications are needed to be performed at each new sample time. When the input signal is a pure sine wave of fundamental frequency i.e. $X_{N+r}=X_{r}, X^{*}{ }^{(r-1)}$ becomes $X^{*(r)}$ for all $r$. Therefore, when a recursive computation is used to calculate phasors, it leads to stationary phasors in the complex plane.

\section{PHASOR MEASUREMENT UNIT MODEL IN MATLAB/SIMULINK}

The developed MATLAB/SIMULINK model of PMU is shown in Fig. 1. Various blocks of MATLAB/SIMULINK model of PMU are explained below.

\section{A. Input Signal (Sine Wave)}

The analog sinusoidal signal is provided as an input to the PMU in the form of sine wave. These analog inputs may be the output of current and voltage transformers.

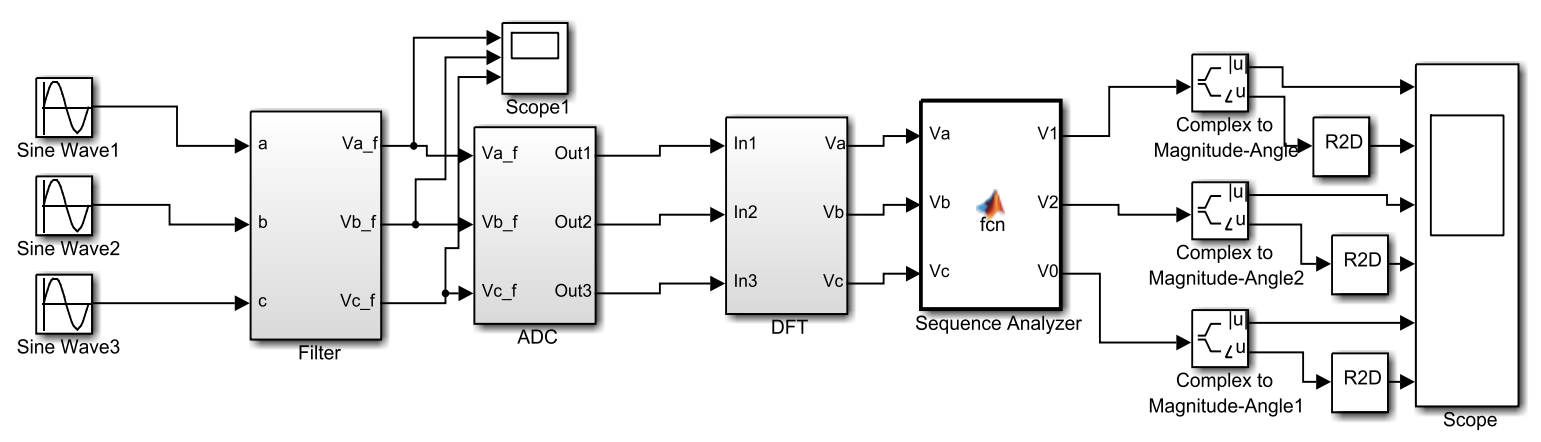

Fig. 1. MATLAB/SIMULINK model of PMU.

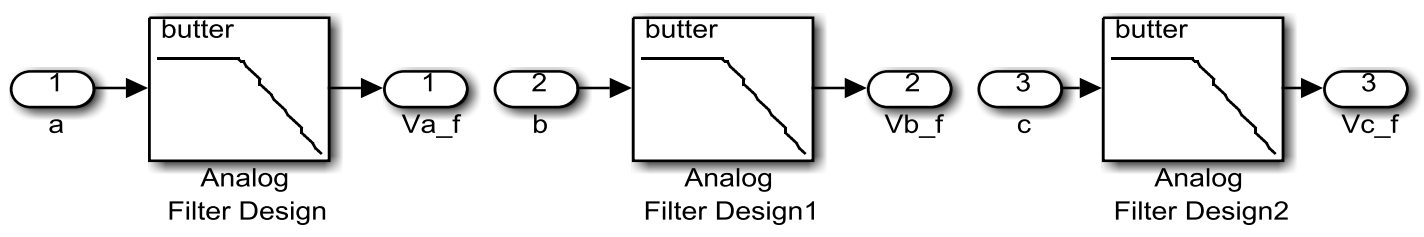

Fig. 2. Butterworth band pass filter of order 2.

\section{B. Filter}

An anti-aliasing filter is used before a signal sampling to satisfy the sampling theorem, and to approximately limit the bandwidth of a signal. The Butterworth Band Pass filter specifically have center frequency $f 0=50 \mathrm{~Hz}$, bandwidth $\Delta f=10 \mathrm{~Hz}$, and has flat response in pass-band as compared to other filter. The Butterworth filter of 2 nd order is chosen to obtain better results. The 2 nd order Butterworth band-pass filter has accurate filter response as compared to 3rd order and 5 th order. The 2 nd order filter response for a signal $x(t)=230(\operatorname{Cos} w 1 t 1+\operatorname{Cos} w 2 t 2+\operatorname{Cos} w 3 t 3)$ having frequency $w 1=40 \mathrm{~Hz}$ for $t=1 \mathrm{~s}, w 2=50 \mathrm{~Hz}$ for $t=0.5 \mathrm{~s}, w 3=60 \mathrm{~Hz}$ for $t=0.5 \mathrm{~s}$ with center frequency $\mathrm{f} 0=50 \mathrm{~Hz}$ and bandwidth $\Delta f=10$ $\mathrm{Hz}$ has been considered in present work and is shown in Fig. 2.

\section{Analog-to-Digital Converter (ADC)}

ADC is a device used to convert a continuous signal to discrete form physical quantity i.e. voltage in terms of discrete form. Quantization of the input involves in ADC, which introduces a small amount of error. ADC consists of pulse generator, quantizer and sample and hold circuit as shown in Fig. 3. The sample \& hold circuit gives the 
equivalent result of an ADC. Sample and hold circuit converts the input signal into digital signal.

In PMU, Global Positioning System (GPS) receiver is used for time stamping. In present work, pulse generator has been used that generates 1000 pulses for sampling rate of 20 samples/cycle in 1s as an input signal and has been sampled in accordance with output of the pulse generator.

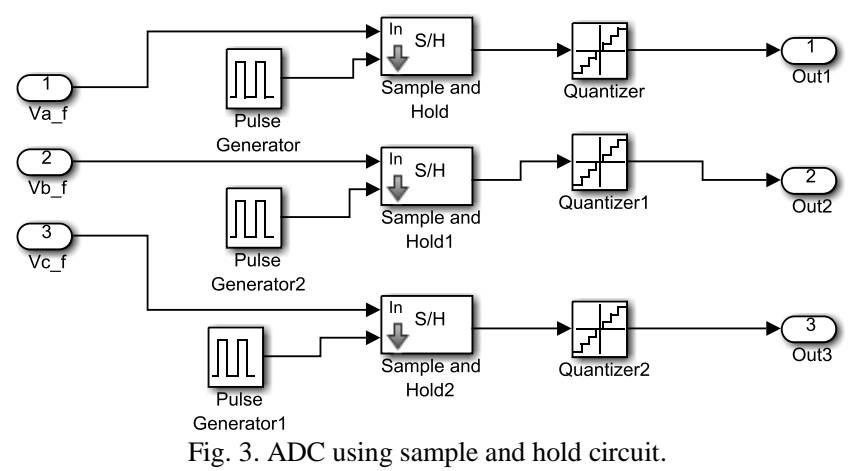

The quantizer is used to round off error and discretize the input at the interval of $0.5 \mathrm{~s}$. This represents Clock Synchronization Unit i.e. used to detect 1 Pulse Per Second (PPS) synchronizing signals from GPS source. A control system that generates an output signal whose phase is related to the phase of an input signal represents Phase Locked Loop (PLL).

\section{Discrete Fourier Transform (DFT)}

This block has been used to estimate phasors using DFT considering non-recursive and recursive algorithms.

\section{E. Sequence Analyzer}

The estimated voltage and current phasors are used as inputs for embedded MATLAB function known as sequence analyzer that helps in obtaining positive sequence phasor.

\section{Phasor Measurement Unit Model in LABVIEW}

Laboratory Virtual Instrument Engineering Workbench (LABVIEW) uses programs i.e. represented by icons to create applications and these programs are called Virtual Instruments (VI). The Graphical Interface (GI) of LABVIEW consists of front and back panel window.

- The inputs are predefined and controlled from the front panel and output is reflected in front panel only;

- Back panel is used to connect VI to construct the logical operations.

LABVIEW models have been constructed using standard library VI, and also using some user defined VI.

In the non-recursive algorithm, the input signal has been generated from library VI i.e. Simulate Signal VI. This analog input signal is then converted to discrete signal with A2D VI and stored in an array. Here, data window is considered for 12 samples. A user defined VI has to be prepared for calculating the Fourier coefficient of the data samples. Appropriate arithmetic operations are performed to estimate phasor for first data window. The complex term obtained after phasor calculation is converted into polar form and displayed as output. This algorithm is repeated for subsequent data samples. As newer estimate of phasor is performed, the phasor rotates anticlockwise by an angle $\theta$ due to delay of each sample by one sampling angle. The developed non-recursive LABVIEW model is shown in Fig. 4.

For recursive algorithm, phasors are to be estimated over a data window. The non-recursive algorithm has been used to generate the first estimate of phasor in the present work. Once the phasor has been obtained for the first data window, recursive algorithm has been used.

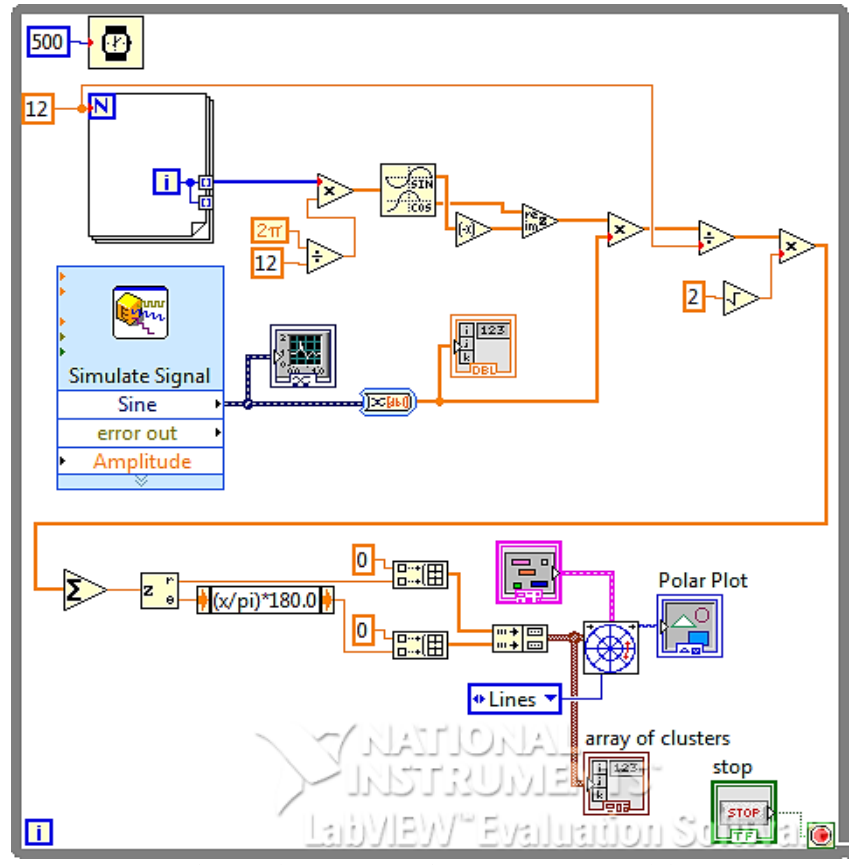

Fig. 4. The non-recursive LABVIEW model.

When phasor estimate is obtained for $(N+r-1)^{\text {th }}$ sample, $(N+r)^{\text {th }}$ sample is compared with $r^{\text {th }}$ sample using suitable mathematical operands provided in the standard library. The developed recursive LABVIEW model for stage I and stage II is shown in Figs. 5(a) and 5(b).

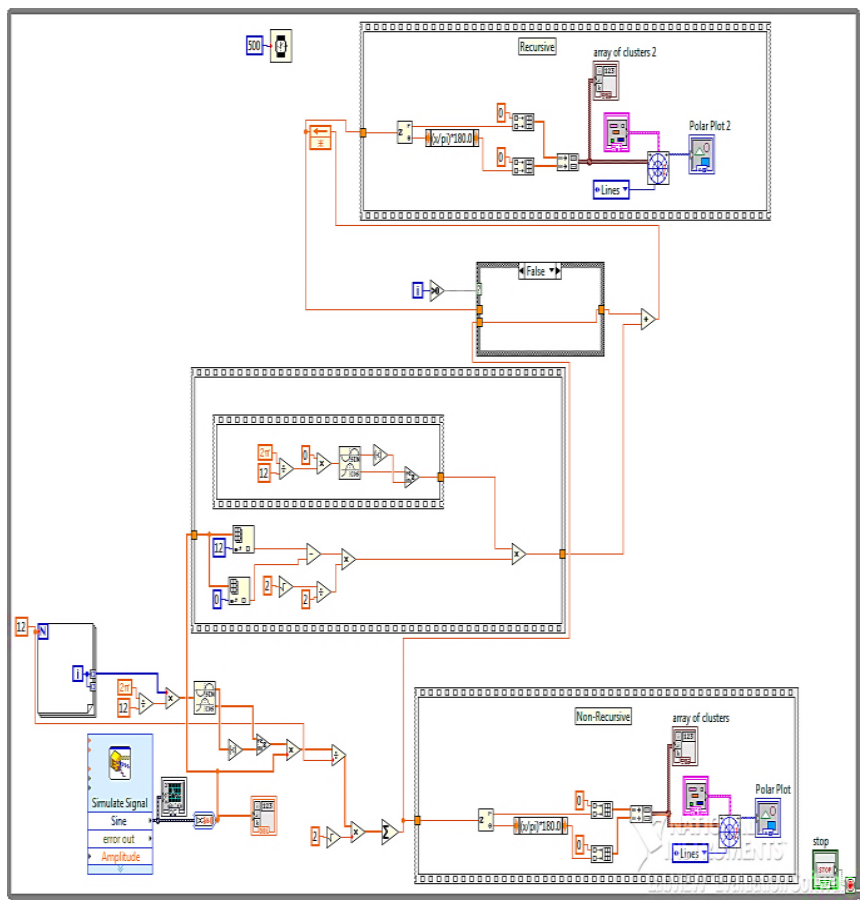

Fig. 5(a). The recursive LABVIEW model-stage I (false condition) 


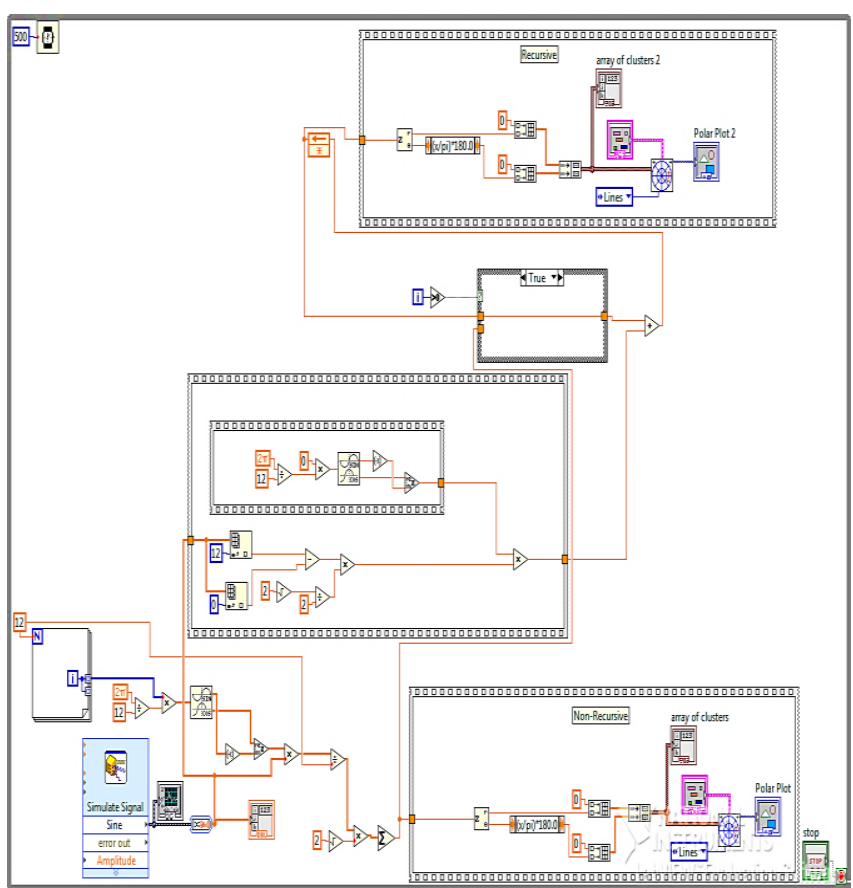

Fig. 5(b). The recursive LABVIEW model- Stage II (True Condition).

\section{CASe Studies}

MATLAB Simulation and LABVIEW model for PMU were developed and compared for recursive and non-recursive DFT algorithms to get the required phase and magnitude of the signal.

A test case have been considered for single phase $230 \mathrm{~V}$, $x(t)=230 \operatorname{Cos}\left(100 \pi t+\frac{\pi}{4}\right)$ at frequency $50 \mathrm{~Hz}$ which is sampled at a sampling frequency of $600 \mathrm{~Hz}$ i.e. 12 samples per cycle. The phasor output in polar form for non-recursive and recursive estimates is shown in Figs. 6(a), 6(b), 6(c), 6(d), respectively. It is observed from the Figs. 6(a) \& 6(b) that phasor estimates obtained with non-recursive algorithm maintains a constant magnitude. However, it is observed from Fig. 6(b) that the new phasor is advanced by $30^{\circ}$ from the phasor shown in Fig. 6(a). It is observed from Figs. 6(c) and 6(d) that with recursive phasor estimation, estimated magnitude and phase of the phasor remains constant over different data windows.

The phasor has been estimated using recursive and non-recursive algorithms. The estimated phasors are shown in Table I. Developed MATLAB based model and LABVIEW based model resulted in same phasor estimation using recursive and non-recursive algorithms. However, computational times in phasor estimation by two models were different. Computational time for phasor estimation using non-recursive and recursive algorithm for the MATLAB based model and LABVIEW based model is shown in Table I. It is observed from Table I that MATLAB based model requires less computational time in phasor estimation as compared to LABVIEW based model using non-recursive and recursive algorithms. It is also observed from Table I that recursive algorithm based phasor estimation takes less computational time compared to non-recursive algorithm for the MATLAB based models for most of the cases, whereas, for the LABVIEW based models, the two algorithms require same computational effort. First 20 samples have been considered for phasor estimation. Since phasor estimation is performed over a cycle, the first phasor is obtained after one complete cycle of the sinusoid i.e. after obtaining 12 samples. Due to this, first 12 columns of Table I of the recursive and non-recursive phasor updates are empty, at which time the first data window is completely filled.

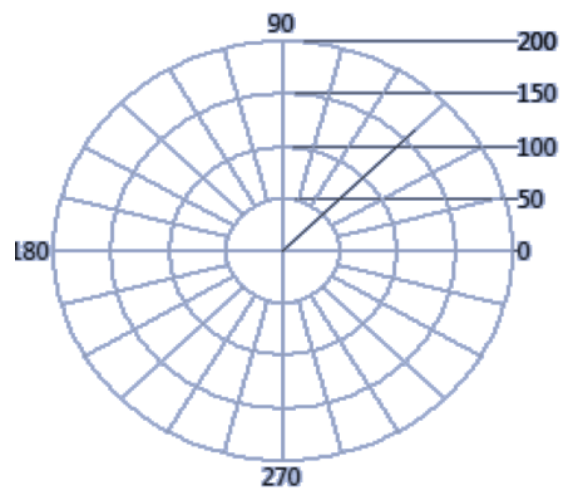

Fig. 6(a). Non-recursive Phasor estimation for first data window (with fixed magnitude and phase angle $=45^{\circ}$ ).

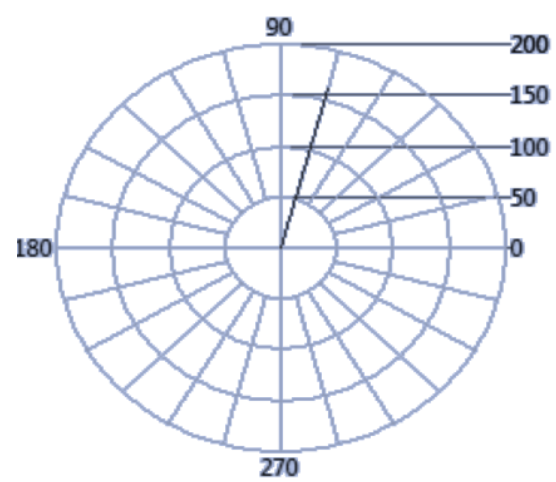

Fig. 6(b). Non-Recursive Phasor Estimation for second data window (with fixed magnitude and phase angle $=75^{\circ}$ ).

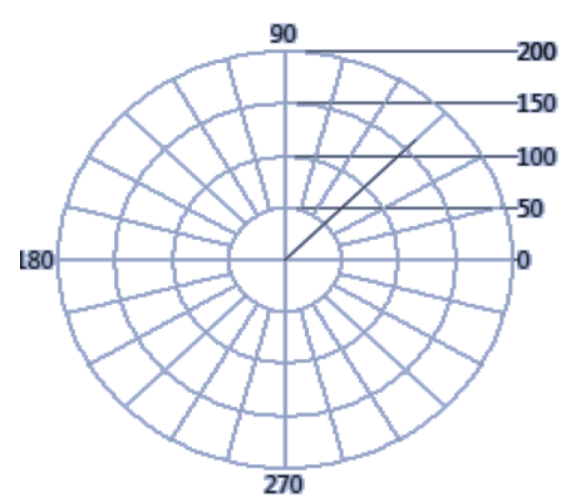

Fig. 6(c). Recursive Phasor estimation for first data window (with fixed magnitude and fixed phase angle $=45^{\circ}$ ).

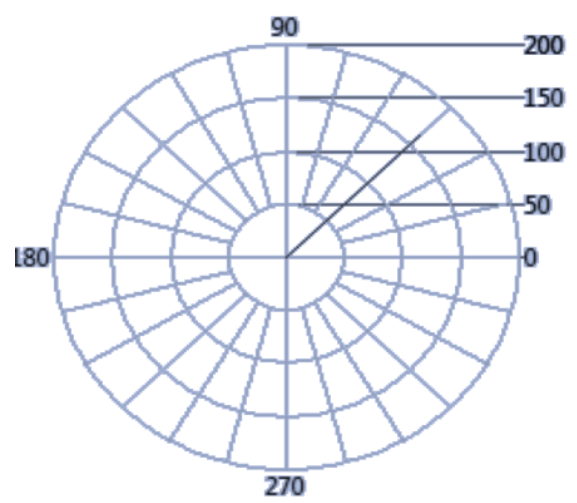

Fig. 6(d). Recursive Phasor Estimation for second data window (with fixed magnitude and fixed phase angle $=45^{\circ}$ ). 
TABLE I: COMPUTATIONAL TIME FOR PHASOR ESTIMATION USING NON-RECURSIVE AND RECURSIVE ALGORITHM FOR THE MATLAB AND LABVIEW

\begin{tabular}{|c|c|c|c|c|c|c|c|}
\hline \multirow{3}{*}{$\begin{array}{l}\text { Sample } \\
\text { No. }\end{array}$} & \multicolumn{4}{|c|}{ Computational Time } & \multirow{3}{*}{ Samples (Xn) } & \multirow{3}{*}{$\begin{array}{c}\text { Non-Recursive } \\
\text { phasor estimates }\end{array}$} & \multirow{3}{*}{$\begin{array}{c}\text { Recursive } \\
\text { phasor } \\
\text { estimates }\end{array}$} \\
\hline & \multicolumn{2}{|c|}{ In MATLAB } & \multicolumn{2}{|c|}{ In LABVIEW } & & & \\
\hline & $\begin{array}{c}\text { In } \\
\text { Non-Recursi } \\
\text { ve Estimation } \\
\text { (Micro-secs.) }\end{array}$ & $\begin{array}{c}\text { In Recursive } \\
\text { Estimation } \\
\text { (Micro-secs.) }\end{array}$ & $\begin{array}{c}\text { In } \\
\text { Non-Recursi } \\
\text { ve Estimation } \\
\text { (Secs.) }\end{array}$ & $\begin{array}{l}\text { In Recursive } \\
\text { Estimation } \\
\text { (Secs.) }\end{array}$ & & & \\
\hline 0 & 19.89 & 16.68 & 0 & 0 & 162.635 & -- & -- \\
\hline 1 & 6.41 & 5.13 & 0.0016 & 0.0016 & 59.5284 & -- & -- \\
\hline 2 & 1.28 & 2.57 & 0.0033 & 0.0033 & -59.5284 & -- & -- \\
\hline 3 & 1.92 & 1.92 & 0.005 & 0.005 & -162.635 & -- & -- \\
\hline 4 & 1.92 & 2.57 & 0.0066 & 0.0066 & -222.163 & -- & -- \\
\hline 5 & 1.28 & 2.57 & 0.0083 & 0.0083 & -222.163 & -- & -- \\
\hline 6 & 1.92 & 3.21 & 0.01 & 0.01 & -162.635 & -- & -- \\
\hline 7 & 1.28 & 2.57 & 0.011 & 0.011 & -59.5284 & -- & -- \\
\hline 8 & 1.92 & 3.21 & 0.013 & 0.013 & 59.5284 & -- & -- \\
\hline 9 & 1.28 & 3.21 & 0.015 & 0.015 & 162.635 & -- & -- \\
\hline 10 & 1.28 & 3.21 & 0.016 & 0.016 & 222.163 & -- & -- \\
\hline 11 & 1.92 & 2.57 & 0.018 & 0.018 & 222.163 & -- & -- \\
\hline 12 & 45.55 & 3.85 & 0.02 & 0.02 & 162.635 & $162.635 \angle 45^{\circ}$ & $162.635 \angle 45^{\circ}$ \\
\hline 13 & 39.77 & 3.85 & 0.021 & 0.021 & 59.5284 & $162.635 \angle 75^{\circ}$ & $162.635 \angle 45^{\circ}$ \\
\hline 14 & 43.62 & 3.85 & 0.023 & 0.023 & -59.5284 & $162.635 \angle 105^{\circ}$ & $162.635 \angle 45^{\circ}$ \\
\hline 15 & 42.34 & 3.85 & 0.025 & 0.025 & -162.635 & $162.635 \angle 135^{\circ}$ & $162.635 \angle 45^{\circ}$ \\
\hline 16 & 39.77 & 3.85 & 0.026 & 0.026 & -222.163 & $162.635 \angle 165^{\circ}$ & $162.635 \angle 45^{\circ}$ \\
\hline 17 & 42.34 & 3.85 & 0.028 & 0.028 & -222.163 & $162.635 \angle-165^{\circ}$ & $162.635 \angle 45^{\circ}$ \\
\hline 18 & 42.34 & 3.21 & 0.03 & 0.03 & -162.635 & $162.635 \angle-135^{\circ}$ & $162.635 \angle 45^{\circ}$ \\
\hline 19 & 41.70 & 3.85 & 0.031 & 0.031 & -59.5284 & $162.635 \angle-105^{\circ}$ & $162.635 \angle 45^{\circ}$ \\
\hline
\end{tabular}

\section{CONCLUSIONS}

In this paper two models have been developed for estimation of phasors using PMUs i.e. (i) MATLAB based model and (ii) LABVIEW based model. Both the models consider DFT with non-recursive as well as recursive algorithms in phasor estimation. Out of the two models, MATLAB based model requires less computational effort compared to LABVIEW based model. Proposed models are quite simple to be developed, and may be effective for research purposes.

\section{REFERENCES}

[1] A. G. Phadke and J. S. Thorp, "Synchronized phasor measurements and their applications," New York, Springer, 2008.

[2] J. D. L. Ree, V. Centeno, J. S. Thorpand, and A. G. Phadke, "Synchronized phasor measurement applications in power systems," IEEE Transactions on Smart Grid, vol. 1, no. 1, June 2010.

[3] V. Krishna, R. S. Ashok, and M. G. Krishnan, "Synchronized phasor measurement unit," IEEE International Conference on Power, Signals, Controls and Computation, Kerala, pp. 1-6, January 2014.

[4] S. V. Hareesh, P. Raja, and M. P. Selvan, "An effective implementation of phasor measurement unit (PMU) by using non-recursive DFT algorithm," in Proc. International Conference on Condition Assessment Techniques in Electrical Systems (CATCON), Banglore, India, pp. 195-199, December 2015.

[5] G. Sanchez-Ayala, J. R. Aguero, D. Elizondo, and M. (Dino) Lelic, "Current trends on applications of PMUs in distribution systems," IEEE Innovative Smart Grid Technologies, Washington, DC, pp. 1-6, February 2013.
[6] D. Ghosh, T. Ghose, and D. K. Mohanta, "Communication feasibility analysis for smart grid with Phasor measurement units," IEEE Transactions on Industrial Informatics, vol. 9, no. 3, pp. 1486-1496, August 2013.

[7] Y. Yang and S. Roy, "PMU placement for optimal three-phase state estimation performance," in Proc. IEEE International Conference on Smart Grid Communications, Vancouver, Canada, pp. 342-347, October 2013.

[8] F. Aminifar, M. Shahidehpour, M. Fotuhi-Firuzabad, and S. Kamalinia, "Power System Dynamic State Estimation with Synchronized Phasor measurements," IEEE Transactions on Instrumentation and Measurement, vol. 63, no. 2, pp. 352-363, February 2014.

[9] D. Dotta, J. H. Chow, L. Vanfretti, M. S. Almas, and M. N. Agostini, "A MATLAB-based PMU simulator," in Proc. IEEE Power and Energy Society General Meeting, pp. 1-5, July 2013.

[10] M. Zhou, V. A. Centeno, J. S. Thorp, and A. G. Phadke, "An alternative for including Phasor measurements in state estimators," IEEE Transactions on Power Systems, vol. 21, no. 4, November 2006.

[11] A. Borghetti, C. A. Nucci, M. Paolone, G. Ciappi and Aurelio, "Synchronized phasors monitoring during the islanding maneuver of an active distribution network," IEEE Transactions on Smart Grid, vol. 2, no. 1, March 2011.

[12] D. R. Gurushinghe, A. D. Rajapakse and D. Muthumuni, "Modeling of a synchrophasor measurement units into an electromagnetic transient simulation program," in Proc. International Conference on Power Systems Transients (ISPT), Vancouver, Canada, July 18-20, 2013.

[13] A. T. Al-Hammouri, L. Nordstrom, M. Chenine, L. Vanfretti, N. Honeth, and R. Leelaruji, "Virtualization of synchronized phasor measurement units within real-time simulators for smart grid applications," in Proc. IEEE Power and Energy Society General Meeting, Canada, pp. 1-7, July 2012.

[14] K. Zhu, S. Deo, A. T Al-Hammouri, N. Honeth, M. Chenine, D. Babazadeh, and L. Nordstrom, "Test platform for synchrophasor based wide-area monitoring and control applications," in Proc. IEEE Power and Energy Society General Meeting, Canada, pp.1-5, July 2013. 


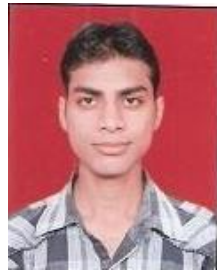

Alok Jain received his B.Tech (Hons.) degree electrical \& electronics engineering (EN) from KCNIT, Banda, U.P., India in 2010 and M.E. degree in electrical engineering (power systems \& electric drives) from Thapar University, Patiala, Punjab, India in 2013. He is currently pursuing for $\mathrm{PhD}$ degree in electrical engineering (power systems) from Indian Institute of Technology (BHU), Varanasi, India. His main research interests include smart grid, micro grid, power quality, distributed generation and renewable energy.

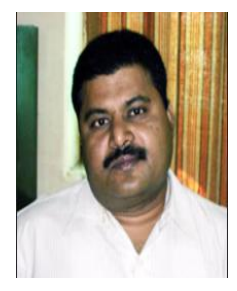

M. K. Verma received his B.Sc. (Engg.) degree from National Institute of Technology (NIT), Rourkela, India and M.Sc. (Engg.) degree from Bihar Institute of Technology (BIT), Sindri, India. He obtained $\mathrm{PhD}$ degree in electrical engineering from Indian Institute of Technology (IIT), Kanpur, India in 2005. He is currently working as a professor in Electrical Engineering Department at Indian Institute of Technology (BHU), Varanasi, India since 2005. His main research interests include voltage stability studies, power quality, smart grid and applications of FACTS controllers. 